\title{
Mycotic aneurysm of left subclavian artery
}

\author{
S K Vyas, N W Law, C A Loehry
}

\begin{abstract}
Bacterial endarteritis is rare and usually affects the abdominal aorta. A case of bacterial left subclavian arteritis complicated by mycotic aneurysm is presented. An early diagnosis of infective arteritis was made based on a history of fever, sweats, rigors, and a progressive macular rash, polyarthralgia splinter haemorrhage (limited to the left arm), and a loud left subclavian bruit on admission. A mycotic aneurysm was diagnosed by computed tomography and treated surgically. In cases of endarteritis the possibility of a mycotic aneurysm should be borne in mind even when there is no evidence of endocarditis.
\end{abstract}

(Br Heart F 1993;69:455-456)

\section{Case report}

A 56 year old man presented with a four day history of fever, rigors, general malaise, and night sweats. He also complained of severe restriction of movement in his left shoulder, elbow, wrist, knee, and the middle two fingers of his left hand. There was no previous history of arthritis, rheumatic fever, or valvar heart disease. He was edentulous and had not undergone any recent dental or surgical procedures.

He had mild chronic stable angina on exertion that required no treatment and intermittent claudication in his left leg on walking 100 yards. He smoked approximately an ounce of tobacco per week.

On examination he was pyrexial $\left(38.8^{\circ} \mathrm{C}\right)$ with two splinter haemorrhages of the nailfolds of his left hand. There was a macular, erythematous rash over his left arm and upper chest. There was severe polyarthritis of the left shoulder, elbow, wrist, and knee with restricted movement in all directions. Numerous tender nodules were present on his left palm. Atrial fibrillation with an uncontrolled ventricular response was noted (120 beats per minute) but the blood pressure was sustained at $140 / 95 \mathrm{mmHg}$. The heart sounds were normal but a loud left subclavian bruit was audible. The left femoral and distal pulses were impalpable with a diminished right femoral pulse. There was no splenomegaly or haematuria and the rest of the physical examination was normal. In particular no potential source of infection was found.

The electrocardiograph confirmed atrial fibrillation with evidence of left ventricular hypertrophy on voltage criteria. The chest radiograph showed bilateral pulmonary venous congestion but a normal cardiac silhouette.

Laboratory investigations were as follows: haemoglobin $134 \mathrm{~g} / \mathrm{l}$, mean corpuscular volume $89.4 \mathrm{fl}$, white blood cell count $6.8 \times$ $10^{9} / 1$, erythrocyte sedimentation rate $88 \mathrm{~mm}$ in the first hour. The urea electrolytes, creatinine, and glucose were normal as was the clotting screen. A provisional diagnosis of acute infective arteritis was made but there was uncertainty about which diseased vessel was involved. Blood cultures grew Staphylococcus aureus sensitive to flucloxacillin and gentamicin and intravenous treatment with these two antibiotics was started.

An echocardiogram showed normal cardiac chambers and valve architecture with no vegetations. A duplex scan showed a $50 \%$ stenosis in the right internal carotid artery and that the left internal carotid artery was occluded at its origin. Digital subtraction angiography showed a left subclavian stenosis (fig 1) and occlusion of the left common iliac artery.

Serology for syphilis (VDRL and TPHA), antinuclear factor, and antineutrophil cytoplasmic antibody was negative and a biopsy specimen of the temporal artery was normal.

After four weeks his clinical condition had improved and so intravenous antibiotics were discontinued. The possibility of a mycotic aneurysm at the site of the infection was considered and therefore thoracic computed tomography was performed. This showed a $5 \mathrm{~cm}$ diameter aneurysm of the left subclavian artery (fig 2) which had developed during four weeks after angiography.

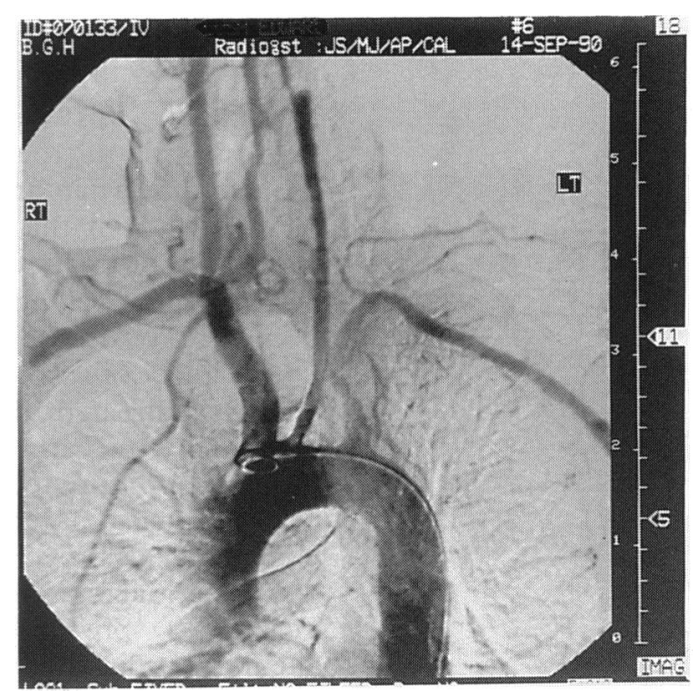

Figure 1 Digital subtraction angiogram of the aortic arch showing left subclavian stenosis.

\author{
Correspondence to \\ Dr S K Vyas, Level D, \\ General Hospital, Tremona \\ Road, Southampton, \\ Hampshire. SO9 4XY \\ Department of
Medicine, Royal \\ Bournemouth General \\ Department of \\ Surgery, Royal \\ Bournemouth Genera \\ Hospital, East Dorset
}


Figure 2 Computed tomogram of the thorax showing mycotic aneurysm of origin of left subclavian artery.

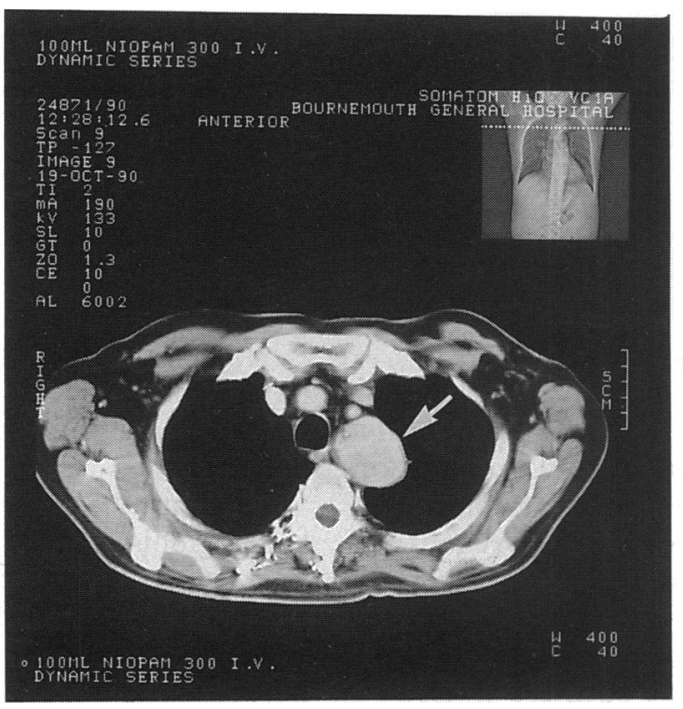

At left thoracotomy a $5 \mathrm{~cm}$ aneurysm of the origin of the left subclavian artery was found which ruptured as the adherent lung was dissected free. The haemorrhage was controlled while the aneurysm was excised and the defect oversewn. A left carotid-axillary reversed vein bypass graft was performed. A culture of the dissected wall of the aneurysm was sterile. Treatment with intravenous flucloxacillin was restarted and he made a good recovery postoperatively. Distal pulses were present and confirmed by Doppler ultrasound in the left arm.

Just before discharge an enhanced thoracoabdominal computed tomogram showed no further evidence of mycotic aneurysm and the patient was discharged three weeks postoperatively. He had had a two week course of intravenous flucloxacillin.

\section{Discussion}

The diagnosis of bacterial infection in the arterial wall is often difficult. This is largely because patients usually present with nonspecific symptoms such as malaise, lethargy, and fever with pain in the region of the involved artery. A palpable aneurysm is present in less than $50 \%$ of patients. ${ }^{1}$ The diagnosis was suspected in this case because of a loud left subclavian artery bruit and the distribution of the rash and polyarthritis. Presumably, this was an effect of dissemination of septic microemboli from the origin of the left subclavian artery. An aspirate of synovial fluid from the left knee, however, was sterile before treatment with antibiotics, suggesting that swelling of this joint may have been reactive. In the absence of specific signs a high index of suspicion must be maintained because untreated, this condition has a poor outcome. An early diagnosis allows both prompt, appropriate antibiotic treatment and timely surgical intervention before invasive pathogens destroy the arterial wall. Predisposing factors to infective endarteritis include pre-existing atherosclerosis, aneurysms, diabetes, immunosuppression, or active vasculitis. The pathogenesis, however, remains unclear in many cases. Atherosclerosis was the most likely predisposing factor in our patient, who had coexisting angina and intermittent claudication. The commonest site of endarterial infection is the abdominal aorta. ${ }^{2}$ Infection of the main intrathoracic arteries is rarely reported and may present as haemoptysis ${ }^{3}$ or may mimic a bronchogenic carcinoma. ${ }^{4}$

Numerous organisms can cause infection in the arterial wall but the most frequent is Salmonella, which is found in $36 \%$ of cases and has a predilection for larger, diseased blood vessels. ${ }^{5}$ Other organisms known to cause such infection are staphylococci, streptococci, Escherichia coli, Pseudomonas, Bacteroides, Haemophilus, Clostridia, and Enterobacter klebsiella. ${ }^{2}$ These were found in 21 patients with abdominal aortic endarteritis in a survey of four American institutions over a ten year period. ${ }^{2}$

Early diagnosis may be aided by greater awareness and improved radiological techniques such as ultrasonography, computer aided tomography, and digital subtraction angiography. In particular enhanced computed tomography may demonstrate changes in the size or appearance of the infected artery or show periarterial nodularity, air, or pus in the arterial wall. ${ }^{2}$ Arteriography is not specific but may show atheromatous disease or a saccular or multiloculated aneurysm in an otherwise normal vessel, suggesting a local infection. ${ }^{6}$ It is important to recognise that an infected artery may rupture before false aneurysmal dilatation has occurred. ${ }^{7}$

The accepted management of bacterial arteritis is intravenous antibiotic therapy, excision and debridement of the artery and the mycotic false aneurysm if present, and where possible, extra-anatomic vascular reconstruction along an uncontaminated path. $^{1}$ In this case extra-anatomic bypass through an uncontaminated field was straightforward and did not compromise the distal circulation. A reversed vein bypass graft was used to reduce the risk of secondary bloodborne infection that is associated with using prosthetic material. ${ }^{8}$

This case shows the importance of early diagnosis in bacterial arteritis. It highlights $N$ the medical and surgical aspects of management while emphasising the need for careful radiological evaluation of the vasculature for a successful outcome.

We thank Mr Simon Parvin for his permission to report this case and Mrs Barbara Thomas for preparing the typescript.

1 Mundth E D, Darling R C, Alvarado R H, et al. Surgical T management of mycotic aneurysms and the complicamanagement of mycotic aneurysms and the complica- $\mathrm{Am}$ tions of infection in vascular reconstructive surgery. Am f Surg 1969;117:460-70

$2 \mathrm{Oz} \mathrm{M} \mathrm{C,} \mathrm{Brener} \mathrm{B} \mathrm{J,} \mathrm{Buda} \mathrm{J} \mathrm{A,} \mathrm{et} \mathrm{al.} \mathrm{A} \mathrm{ten} \mathrm{year} \mathrm{experience}$ with bacterial aortitis. 7 Vasc Surg 1989;10:439-49.

3 Boundy K, Bignold LP. Syphylitic aneurysm of the right subclavian artery presenting with haemoptysis. Aust NZ f Med 1987;17:533-5.

4 Paradowski LJ, Hajdu I, Coli L, Loewen G. Infected aortitis masquerading as bronchogenic carcinoma. $N Y$ State $₹$ Med 1990;90:415-6.

5 Reddy D J, Ernst C B. Infected aneurysms. In: Rutherford R B, ed. Vascular Súrgery. 3rd edition. London: W B Saunders, 1989; 983-96

6 Weintraub R A, Abrams H.L. Mycotic aneurysm. Am $\mathcal{f}$ Roentgen 1968;102:354-62.

7 Cook A M, Christopoiulos D. Rupture of a non-aneurysmal Salmonella infected aorta. Clin Radiol 1989;40: 605-6.

8 Johansen K, Rusch V, Coffin R. Extra-anatomic bypass for aortic sepsis: a cautionary note. Vasc Surg 1987;16: 59-63. 